\title{
Sexually Transmitted Diseases
}

\section{Sender and receiver acceptability and usability of an online partner notification tool for STI in the Netherlands

\author{
--Manuscript Draft--
}

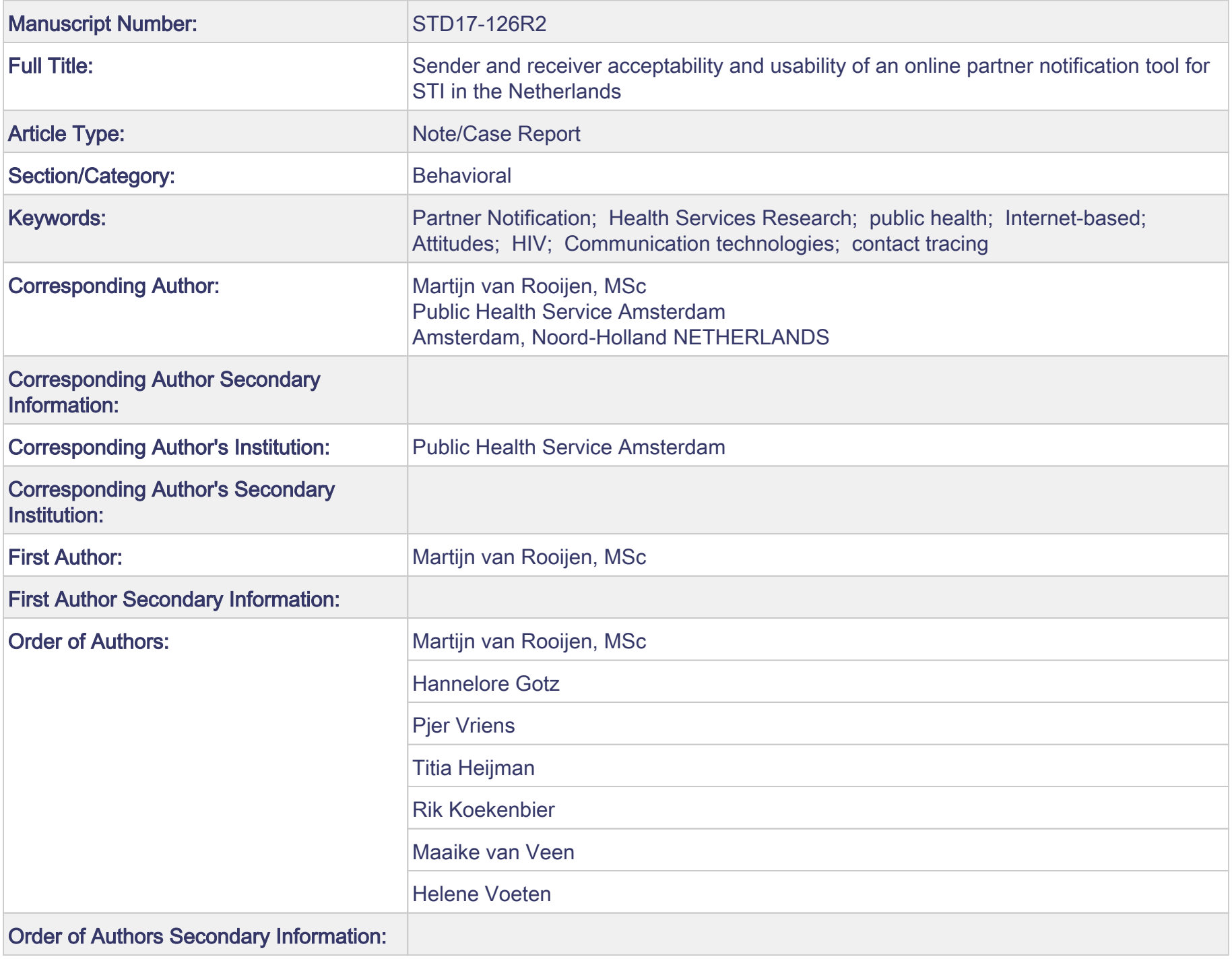




\section{Sexually Transmitted Diseases}

\section{Acknowledgment Form}

Manuscript title:

Sender and receiver acceptability and usability of an online partner notification tool for STI in the Netherlands

Authors must obtain written permission from all individuals who are listed in the Acknowledgments section of the manuscript, because readers may infer their endorsement of data and conclusions. The corresponding author must sign the Acknowledgment form and submit online with the manuscript. The corresponding author must sign the following statement, certifying that (1) all persons who have made substantial contributions in the manuscript (eg. Data collection, analysis, or writing or editing assistance), but who do not fulfill authorship criteria, are named in the Acknowledgements section of the manuscript; (2) all persons named in the Acknowledgments section have provided the corresponding author with written permission to be named in the manuscript; and (3) if an Acknowledgments section is not included, no other persons have made substantial contributions to this manuscript.

Corresponding Author Signature:

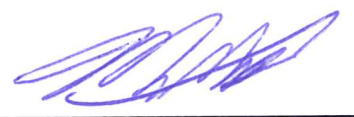

Date: 6-04-2017 
To the Editor-in-Chief, Dr. William C. Miller, MD, Sexually Transmitted Diseases

Amsterdam, 27 September, 2017

Dear Dr. Miller,

Ref.: Ms. No. STD17-126

Title: Sender and receiver acceptability and usability of an online partner notification tool for STI in the Netherlands

Thank you very much for your email of 25 September 2017 accepting our manuscript (STD17-126) for publication after preparing one minor revision.

Hereby we would like to submit our revised version that has been adjusted. This letter includes our response to the comment of the reviewer; below you will find the original comment $(C)$ and our response $(R)$.

Sincerely yours,

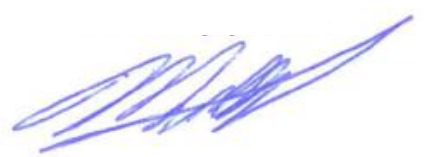

Martijn van Rooijen, on behalf of all authors 


\section{Authors response to the reviewer comment}

C: The one remaining concern I have is that the website "sugestatest.nl" is no longer active and may confuse the readers who may miss the explanation of name change to "partnerwaarschuwing.nl". This confusion may be compounded by the prominent use of the former site name throughout the paper. Perhaps better would be to use the new name with an English translation and a comment about the name change at first mention. The name change also begs the questions why the change was made? $\mathbf{R}$ : We agree that mentioning the name change in the introduction without any explanation is confusing. As the evaluation in our manuscript concerned the tool Suggestatest.nl (the name was changed after the inclusion period) we think that using this name throughout the manuscript is appropriate.

We have omitted the name change from the introduction (line 68). In the second last paragraph of the discussion, we added a sentence mentioning the name change (including English translation of Partnerwaarschuwing.nl) and explained the reason why the name was changed (lines 242-244).

Original sentence in the introduction:

To date, Suggestatest.nl (nowadays called "Partnerwaarschuwing.nl") and CheckOUT (Portugal) are to our knowledge the only published health care provider initiated Internet-based notification systems that are designed for patients with a verified STI only.

Revised sentence in the introduction:

To date, Suggestatest.nl and CheckOUT (Portugal) are to our knowledge the only published health care provider initiated Internet-based notification systems that are designed for patients with a verified STI only.

Added sentence to the discussion:

After the inclusion period of this study, the tool was renamed to "Partnerwaarschuwing.nl" (Partnernotification.nl in English) as some notified partners reported that were confused about the name Suggestatest.nl. 
1 SENDER AND RECEIVER ACCEPTABILTY AND USABILTY OF AN ONLINE

2 PARTNER NOTIFICATION TOOL FOR STI IN THE NETHERLANDS

3

4 Martijn S. van Rooijen ${ }^{1}, \mathrm{MSc}$, Hannelore Gotz ${ }^{2,3}, \mathrm{MD}, \mathrm{PhD}$, Pjer Vriens ${ }^{2}$, Titia Heijman ${ }^{1}$,

$5 \quad \mathrm{PhD}$, Rik Koekenbier ${ }^{1}, \mathrm{MSc}$, Maaike van Veen ${ }^{1}, \mathrm{PhD}$, Helene Voeten ${ }^{2,3}, \mathrm{PhD}$

6

$7 \quad{ }^{1}$ Public Health Service of Amsterdam (GGD Amsterdam), Department of Infectious Diseases,

8 the Netherlands

$9 \quad{ }^{2}$ Department Infectious Disease Control, Public Health Service Rotterdam-Rijnmond, the

10 Netherlands

$11{ }^{3}$ Department of Public Health, Erasmus MC, Rotterdam, the Netherlands

12

13 Correspondence to Martijn van Rooijen, Public Health Service of Amsterdam, Department of

14 Infectious Diseases, STI clinic, P.O. Box 2200, 1000 CE Amsterdam, the Netherlands

15 Tel: +31-20-5555874; Fax: +31-20-5555533.

16 Email: mvrooijen@ggd.amsterdam.nl 
17 Word count summary $30(\max 30)$

18 Word count abstract: currently 48 (max 50)

19 Word count body of text: currently $2008(\max 2000)$

20 Number of Tables: 5; 2 in manuscript and 3 as supplement

21 Number of Figures: 1; 0 in manuscript and 1 as supplement

22 Number of references: 15 (30 max)

23

24 Conflicts of Interest:

$25 \quad$ None declared.

27 Funding:

28 This study was supported by the Dutch AIDS foundation (Grant number: 2009085) and the 29 Research and Development Fund of the Public Health Service of Amsterdam.

\section{Running title:}

32 Acceptability and usability of an online partner notification tool for STI

\section{Short Summary:}

- Patients and sex partners rated the online partner notification tool Suggestatest.nl acceptable and usable.

- To use Suggestatest.nl to notify/get notified of HIV was rated less acceptable and usable. 
39 Abstract

40

41 Users (index patients with a verified STI and notified partners) rated the health care provider

42 initiated Internet-based partner notification application Suggestatest.nl acceptable and usable.

43 Both groups were less positive about Suggestatest.nl to notify /get notified of HIV than of

44 other STI. An anonymous notification was perceived less acceptable.

45

46 Keywords:

47 Partner notification, Health Services Research, Public Health, Internet-based, Attitudes, HIV, 48 Communication technologies, Contact tracing

49 
Partner notification (PN) is the process whereby the sexual partner(s) of a patient diagnosed

with a sexually transmitted infection (STI) are identified and informed of their exposure to an

STI. ${ }^{1}$ Many studies show a preference to notify partners face-to-face or by telephone rather

than with technologies like SMS or email. ${ }^{2-5}$ However, internet-based PN might be an additional method to reach more partners. ${ }^{2}$

To assist PN at the STI clinics of Rotterdam and Amsterdam, the Netherlands, an online tool called Suggestatest.nl was developed explicitly for patients who were diagnosed with an STI or HIV infection. Using this tool, index patients could send an anonymous or non-anonymous notification message by email, SMS, postal mail or - with the username of their partner - to a gay social network account. A general evaluation of the use of Suggestatest.nl showed that this novel tool suits a small number of index clients, mainly by sending anonymous text messaging. ${ }^{6,7}$ Out of those intending to use Suggestatest.nl, $23 \%$ notified a partner through Suggestatest.nl and 58\% of the partners notified through Suggestatest.nl logged-in to read their notification online. To date, Suggestatest.nl and CheckOUT (Portugal) are to our knowledge the only published health care provider initiated Internet-based notification systems that are designed for patients with a verified STI only. ${ }^{8}$ Less is known about the acceptability of these tools for both the sender (index patient) and the receiver (notified partner). In addition, much of the published acceptability research relied on hypothetical scenarios of accessing options for PN. ${ }^{9}$ In this study we evaluated the acceptability and usability of Suggestatest.nl in both index patients and notified partners who have used this PN tool. 
Materials and Methods

Study setting

The STI outpatient clinics of Rotterdam and Amsterdam perform respectively about 12,500 and 40,000 STI consultations annually, free of charge and anonymous. In case an STI is diagnosed, the health care professional discusses the PN options and registers the patient's preference. These options consist of patient referral (supported with a contact card or - from March 2012 onward - with Suggestatest.nl), provider referral or contract referral.

\section{Suggestatest.NL}

Patients with a confirmed STI diagnosis (chlamydia, lymphogranuloma venereum, gonorrhea, syphilis, HIV and/or trichomoniasis) received a nurse-generated code when they preferred to use Suggestatest.nl for PN. To notify, the index patient had to login to Suggestatest.nl using the nurse-generated code. For each partner, the patient had to select the method (SMS/email/postal/gay dating site) and the mode (anonymous/non-anonymous) of sending the notification. All partners - irrespective of the above selected method - received a standardized message with a unique partner code and had to login to the website to read about the notified STI or HIV, possible treatment and how to make an appointment at the STI clinic.

Theoretical framework from the "Technology Acceptance Model" (TAM) was used to develop the questionnaires for index patients and notified partners. ${ }^{10}$ The two factors that determine TAM are "perceived usefulness" (referred to as acceptability) and "perceived ease of use" (referred to as usability). ${ }^{10}$ Questionnaires on acceptability and usability to notify/be notified through Suggestatest.nl of STI and HIV were offered online to all participants regardless their diagnosis/received notification. After the index patient had sent a 
101 Suggestatest.nl notification, an invitation window popped-up to complete an online

102 questionnaire. Partners were recruited for an online questionnaire after reading their STI

103 notification online. After completing the questionnaire, participants were asked to fill-in their

104 email address to receive an additional online questionnaire after 2 weeks. The online

105 questionnaires were collected from March 2012 until June 2013 (Supplementary Tables 1 \&

106 2). Because the online response of partners was low, partners visiting the STI clinics and

107 notified through Suggestatest.nl (who had not yet filled-in an online questionnaire) were

108 recruited from July 2012 until June 2013 to fill in a paper-and-pencil questionnaire.

109

$110 \quad$ Statistical analysis and data collection

111 All questionnaire data were analyzed in IBM SPSS Statistics version 21 (IBM Corporation,

112 Armonk, New York, USA). The acceptability and usability scores were constructed from the

113 mean of the items included. Constructs were only calculated if none of the items for this

114 construct had a missing values. For each construct, the reliability was calculated using the

115 Spearman-Brown statistic (2-items) or the Cronbach's coefficient alpha ( $\alpha$ ) (3 or more

116 items). ${ }^{11}$ Reliability values of $\geq 0.7$ were assumed acceptable and all were 0.75 or above.

117 Frequency of Internet use for arranging personal matters was categorized in less frequent

118 (scores 1-3) and frequent (scores 4-5). Respondents and non-respondents were compared with

119 the chi-squared test or Fisher's exact test and the Mann-Whitney U test. Using the

120 Independent $t$ test, the mean scores of notified partners who responded to the online and those

121 who responded to the paper-and-pencil questionnaires, were compared. The paired t-test was

122 used to compare scores on different items within the same group. P values of less than 0.05

123 were considered statistically significant.

124

125 Ethics 
126 This study was waived by the Medical Ethical Committee of the Erasmus University of

127 Rotterdam, because Suggestatest.nl was an extension of standard care.

128 


\section{Index patients}

132 During the study period, $112(19.8 \%)$ out of 565 Suggestatest.nl users completed the

133 questionnaire (Supplementary Figure 1). Response was higher among MSM (27.7\%),

134 compared to heterosexual men $(13.1 \%)$ and women $(17.0 \%, \mathrm{p}=0.002)$, and responders

135 notified a higher median number of partners than non-responders (Supplementary Table 3).

136 Four responders were newly diagnosed with HIV.

138 The majority of index patients reported that they were able to notify more partners than

139 without the existence of Suggestatest.nl (Table 1). The acceptability and usability to use

140 Suggestatest.nl to notify sexual partners of HIV was rated significantly less acceptable and

141 usable (3.0 and 3.6, respectively) than notifying of another STI (4.4 and 4.7, respectively;

$142 \mathrm{p}<0.001$; Table 1). Among MSM, the overall acceptability was higher (4.4) than among non-

143 MSM (4.1; $\mathrm{p}=0.007)$ whereas the overall usability was not different (4.5 versus 4.4,

144 respectively; $\mathrm{p}=0.28$ ).

146 Notified partners

147 Out of 2,030 notified partners, $163(8.0 \%)$ responded to the questionnaires (53 online and 110

148 offline at the STI clinic) (Supplementary Figure 1). Notified partners who filled-in the

149 questionnaire were comparable to those who did not respond (Supplementary Table 1). The

150 acceptability and usability scores of online and offline responders were not significantly

151 different. Of the 106 partners who were notified of HIV exposure, three responded to a 152 questionnaire. 
154 Most notified partners preferred to receive a non-anonymous notification via SMS (Table 2).

155 Partners who were notified anonymously rated their notification less acceptable (2.7) than

156 partners who were notified by name $(4.4 ; \mathrm{p}<0.001)$ (Table 2$)$. The acceptability and usability

157 to be notified of HIV through Suggestatest.nl was rated significantly less acceptable and

158 usable (3.3 and 3.2, respectively) than being notified of another STI (both 4.4; $\mathrm{p}<0.001$ ). The

159 overall acceptability and usability scores of Suggestatest.nl (4.1) did not differ between MSM

160 and non-MSM $(\mathrm{p}=0.28$ and $\mathrm{p}=0.50)$.

161

162

163 


\section{Statement of principal findings}

167 The online PN tool Suggestatest.nl was rated acceptable and usable by both senders (index

168 patients) and receivers (notified partners). Both groups were less positive about

169 Suggestatest.nl to notify /get notified of HIV than of another STI. Partners notified

170 anonymously perceived their mode of notification less acceptable than those notified by

171 name.

172

\section{Strengths and weaknesses of the study}

174 While most papers on acceptability of electronic PN relied on hypothetical scenarios, we

175 measured acceptability and usability in a real setting, in both patients and partners who used

176 Suggestatest.nl. ${ }^{9}$ Moreover, we measured the opinion of both MSM and heterosexuals who

177 used Suggestatest.nl. Patients who chose to use Suggestatest.nl may be more enthusiastic

178 about Suggestatest.nl than STI patients in general. However, their partners who did not have

179 any choice in the method of how they received a notification, were also generally positive

180 about Suggestatest.nl.

181

182 For our study, we recruited notified partners when they visited the website to read their

183 notification or during the resulting consultation at the STI clinic. Unfortunately, the overall

184 participation rate of notified partners was low (8\%). This might have resulted in

185 overestimated acceptability and usability scores, making it difficult to generalize the

186 measured opinion to the general STI clinic population. Due to missing notification codes of

18743 notified clients, no information of the received notification was known. 
The questions concerning the acceptability and usability of using Suggestatest.nl to notify of

190 HIV exposure were mainly answered by patients and partners who notified or were notified of an STI other than HIV. As a consequence, the lower acceptability and usability to notify of

192 HIV through Suggestatest.nl were mainly hypothetical. Theoretically, the usability to notify

193 partners of STI or HIV exposure through Suggestatest.nl should be comparable because it

194 uses the same system with identical actions. However, the construct of usability was rated

195 lower for HIV than other STI, indicating that it probably did not measure usability only.

\section{Comparison with other studies}

198 A study among Peruvian MSM and transgender women diagnosed with STI showed that the

199 introduction of a hypothetical Internet-based PN system resulted in a dramatic increase in

200 anticipated notification of secondary partners. ${ }^{12}$ In our study, almost $80 \%$ of the index patients reported that they had notified more partners than they would have done without the existence of Suggestatest.nl.

A study among Spanish MSM of their anticipated notification behavior showed that face to face or a phone call were the preferred methods to notify of STI or HIV for both stable and casual partners. ${ }^{13}$ An identifiable SMS was the next most popular method to notify stable and casual partners of STI or HIV. The preference for sending an identifiable SMS contradicts our findings: most patients notified their partners anonymously. ${ }^{6,7}$ A similar effect was seen in a

209 UK study: the preference of respondents for a partner notification method was dependent on 210 whether they see themselves as index patients or contacts. ${ }^{14}$ Another possibility is that 211 patients in our study who were willing to send an identifiable SMS or email have used their

212 own mobile or email and only those with interest in sending an anonymous notification have 213 used Suggestatest.nl. 
215 In a review of the acceptability of electronic PN a pattern emerged across studies showing 216 that anonymity was less acceptable than the electronic delivery method itself. ${ }^{9}$ In our study, 217 the same effect was seen: notified partners were less positive about the fact that their 218 Suggestatest.nl notification was anonymous but were still content about Suggestatest.nl.

\section{Implications for clinicians and policymakers}

221 It seems that, according to the opinion of our patients, STI clinics should offer an online PN tool like Suggestatest.nl. As stated by Hottes et al, a web-based PN service like inSPOT should be supplementary to traditional PN tools. ${ }^{15}$ After developing a PN website, the costs of facilitating online PN are relatively low and it can easily be offered as an addition to already existing traditional tools. Based on our findings we would recommend to incorporate the possibility to notify anonymously.

Patients could be asked to immediately start filling in the contact information of their partners in Suggestatest.nl when they are at the STI clinic for a treatment consultation. Possibly,

230 patients are then more motivated to notify their partners than later at home, and public health nurses could assist with this process. However, it is also important to offer Suggestatest.nl use at a later stage, because at the STI clinic the majority of participants reported that they were unable to fill in contact details of all partners.

\section{Unanswered questions and future research}

236 We recognize that there is a possible trade-off between reaching more partners by the 237 implementation of a low threshold online PN tool and the quality of the sent notification: as 238 many partners do not read their online notification (42\%; e.g. because they think it is an 
239 unsolicited message/spam), the sent notification might not have resulted in health care

240 seeking. ${ }^{6}$ Future research should focus on the most suitable ways of directing online notified

241 partners into care. After the inclusion period of this study, the tool was renamed to

242 "Partnerwaarschuwing.nl" (Partnernotification.nl in English) as some notified partners

243 reported that were confused about the name Suggestatest.nl.

244

245 Our study mainly focusses on patients who chose to use Suggestatest.nl and their partners in 246 which participation was low. For generalizability, more research is necessary which measures 247 the opinion of all notified STI-clinic clients regarding online partner notification. 
249 MvR, and RK designed the study protocol, supported by HG, TH, and HV. PV was

250 responsible for the development of the Suggestatest.nl website and the implementation of the 251 online questionnaires. MvR performed the statistical analyses supported by $\mathrm{HG}, \mathrm{MvV}$, and 252 HV. MvR, HG and HV drafted the paper, all authors commented on draft versions, and all 253 approved the final version.

Previously presented:

256 Information from this paper has been disseminated during a poster presentation at the STI \& 257 AIDS World Congress (17 July 2013, Vienna, Austria; abstract number P5.003).

\section{Changed affiliations:}

260 Maaike G. van Veen: Rutgers, Expert Centre for Sexual and Reproductive Health and Rights, 261 P.O. Box 9022, 3506 GA, Utrecht, the Netherlands

\section{Correspondence and requests for reprints to:}

264 Martijn van Rooijen, Public Health Service of Amsterdam, Department of Infectious

265 Diseases, Department of Research, P.O. Box 2200, 1000 CE Amsterdam, the Netherlands

266 Tel: +31-20-5555874; Fax: +31-20-5555533.

267 Email: mvrooijen@ggd.amsterdam.nl

\section{Acknowledgements}

270 The authors would like to thank all the nurses working on this project and all the participants

271 of this study. Special thanks goes to Francine van de Heuvel and Mariette Hamers for

272 coordinating the implementation at the STI clinics and distributing the offline sex partner 
273 questionnaires. We would like to thank Udi Davidovich for his suggestions on analysis of the 274 measured questionnaire data.

275 
1. European Centre for Disease Prevention and Control. Public health benefits of partner notification for sexually transmitted infections and HIV. Stockholm: ECDC; 2013. Available at: http://ecdc.europa.eu/en/publications/Publications/Partner-notification-forHIV-STI-June-2013.pdf. Accessed 29 March 2017.

2. Bilardi JE, Fairley CK, Hopkins CA et al. Experiences and outcomes of partner notification among men and women recently diagnosed with Chlamydia and their views on innovative resources aimed at improving notification rates. Sex Transm Dis 2010; 37:253-258.

3. Hopkins CA, Temple-Smith MJ, Fairley CK et al. Telling partners about chlamydia: how acceptable are the new technologies? BMC Infect Dis 2010; 10:58.

4. Reed JL, Huppert JS, Gillespie GL et al. Adolescent patient preferences surrounding partner notification and treatment for sexually transmitted infections. Acad Emerg Med 2015; 22:61-66.

5. Rietmeijer CA, Westergaard B, Mickiewicz TA et al. Evaluation of an online partner notification program. Sex Transm Dis 2011; 38:359-364.

6. Gotz HM, van Rooijen MS, Vriens P et al. Initial evaluation of use of an online partner notification tool for STI, called 'suggest a test': a cross sectional pilot study. Sex Transm Infect 2014; 90:195-200.

7. Correction. Sex Transm Infect 2015; 91:74.

8. Rocha M, Guerreiro R, Pinto $\mathrm{N}$ et al. Digital partner notification service at a community-based voluntary counselling and testing centre for men who have sex with men: CheckpointLX, Lisbon, Portugal. International AIDS Conference, At Durban, South Africa 2016; AIDS 2016, Available at: http://programme.aids2016.org/Abstract/Abstract/3702. Accessed 29 March 2017.

9. Pellowski J, Mathews C, Kalichman MO et al. Advancing Partner Notification Through Electronic Communication Technology: A Review of Acceptability and Utilization Research. J Health Commun 2016; 21:629-637.

10. Davis, F. D. "Perceived Usefulness, Perceived Ease of Use, and User Acceptance of Information Technology," MIS Quarterly (13:3), 1989, pp. 319-339.

11. Eisinga R, Grotenhuis M, Pelzer B The reliability of a two-item scale: Pearson, Cronbach, or Spearman-Brown? Int J Public Health 2013; 58:637-642.

12. Clark JL, Segura ER, Perez-Brumer AG et al. Potential impact and acceptability of Internet partner notification for men who have sex with men and transgender women recently diagnosed as having sexually transmitted disease in Lima, Peru. Sex Transm Dis 2014; 41:43-45.

13. Carnicer-Pont D, Barbera-Gracia MJ, Fernandez-Davila P et al. Use of new technologies to notify possible contagion of sexually-transmitted infections among men. Gac Sanit 2015; 29:190-197. 
14. Apoola A, Radcliffe K, Das S et al. Preferences for partner notification method: variation in responses between respondents as index patients and contacts. Int J STD AIDS 2007; 18:493-494.

15. Hottes TS, Gilbert M Evaluation of online partner notification services like inSPOT requires starting with the client, not the clinic. Sex Transm Dis 2012; 39:348. 


\section{SENDER AND RECEIVER ACCEPTABILTY AND USABILTY OF AN ONLINE}

2 PARTNER NOTIFICATION TOOL FOR STI IN THE NETHERLANDS

3

4 Martijn S. van Rooijen ${ }^{1}, \mathrm{MSc}$, Hannelore Gotz ${ }^{2,3}, \mathrm{MD}, \mathrm{PhD}$, Pjer Vriens ${ }^{2}$, Titia Heijman ${ }^{1}$,

$5 \quad \mathrm{PhD}$, Rik Koekenbier ${ }^{1}, \mathrm{MSc}$, Maaike van Veen ${ }^{1}, \mathrm{PhD}$, Helene Voeten ${ }^{2,3}, \mathrm{PhD}$

6

$7{ }^{1}$ Public Health Service of Amsterdam (GGD Amsterdam), Department of Infectious Diseases,

8 the Netherlands

$9 \quad{ }^{2}$ Department Infectious Disease Control, Public Health Service Rotterdam-Rijnmond, the

10 Netherlands

$11{ }^{3}$ Department of Public Health, Erasmus MC, Rotterdam, the Netherlands

12

13 Correspondence to Martijn van Rooijen, Public Health Service of Amsterdam, Department of

14 Infectious Diseases, STI clinic, P.O. Box 2200, 1000 CE Amsterdam, the Netherlands

15 Tel: +31-20-5555874; Fax: +31-20-5555533.

16 Email: mvrooijen@ggd.amsterdam.nl 
17 Word count summary $30(\max 30)$

18 Word count abstract: currently 48 (max 50)

19 Word count body of text: currently $1982 \underline{2008}(\max 2000)$

20 Number of Tables: 5; 2 in manuscript and 3 as supplement

21 Number of Figures: 1; 0 in manuscript and 1 as supplement

22 Number of references: 15 (30 max)

23

24 Conflicts of Interest:

25 None declared.

27 Funding:

28 This study was supported by the Dutch AIDS foundation (Grant number: 2009085) and the 29 Research and Development Fund of the Public Health Service of Amsterdam.

31 Running title:

32 Acceptability and usability of an online partner notification tool for STI

\section{Short Summary:}

- Patients and sex partners rated the online partner notification tool Suggestatest.nl acceptable and usable.

- To use Suggestatest.nl to notify/get notified of HIV was rated less acceptable and usable. 
39 Abstract

40

41 Users (index patients with a verified STI and notified partners) rated the health care provider

42 initiated Internet-based partner notification application Suggestatest.nl acceptable and usable.

43 Both groups were less positive about Suggestatest.nl to notify /get notified of HIV than of

44 other STI. An anonymous notification was perceived less acceptable.

45

46 Keywords:

47 Partner notification, Health Services Research, Public Health, Internet-based, Attitudes, HIV, 48 Communication technologies, Contact tracing

49 
Partner notification (PN) is the process whereby the sexual partner(s) of a patient diagnosed

with a sexually transmitted infection (STI) are identified and informed of their exposure to an

STI. ${ }^{1}$ Many studies show a preference to notify partners face-to-face or by telephone rather

than with technologies like SMS or email. ${ }^{2-5}$ However, internet-based PN might be an additional method to reach more partners. ${ }^{2}$

To assist PN at the STI clinics of Rotterdam and Amsterdam, the Netherlands, an online tool called Suggestatest.nl was developed explicitly for patients who were diagnosed with an STI or HIV infection. Using this tool, index patients could send an anonymous or non-anonymous notification message by email, SMS, postal mail or - with the username of their partner - to a gay social network account. A general evaluation of the use of Suggestatest.nl showed that this novel tool suits a small number of index clients, mainly by sending anonymous text messaging. ${ }^{6,7}$ Out of those intending to use Suggestatest.nl, $23 \%$ notified a partner through 65 Suggestatest.nl and 58\% of the partners notified through Suggestatetst.nl logged-in to read 66 their notification online. To date, Suggestatest.nl (nowadays called "Partnerwaarschuwing.nl") and CheckOUT (Portugal) are to our knowledge the only published health care provider initiated Internetbased notification systems that are designed for patients with a verified STI only. ${ }^{8}$ Less is

71 known about the acceptability of these tools for both the sender (index patient) and the receiver (notified partner). In addition, much of the published acceptability research relied on hypothetical scenarios of accessing options for PN. ${ }^{9}$ 
74 In this study we evaluated the acceptability and usability of Suggestatest.nl in both index

75 patients and notified partners who have used this PN tool.

76 
Materials and Methods

78

79

80

81

82

83

\section{$\underline{\text { Study setting }}$}

\section{$\underline{\text { Suggestatest.NL }}$}

The STI outpatient clinics of Rotterdam and Amsterdam perform respectively about 12,500 and 40,000 STI consultations annually, free of charge and anonymous. In case an STI is diagnosed, the health care professional discusses the PN options and registers the patient's preference. These options consist of patient referral (supported with a contact card or - from March 2012 onward - with Suggestatest.nl), provider referral or contract referral.

Patients with a confirmed STI diagnosis (chlamydia, lymphogranuloma venereum, gonorrhea, syphilis, HIV and/or trichomoniasis) received a nurse-generated code when they preferred to use Suggestatest.nl for PN. To notify, the index patient had to login to Suggestatest.nl using the nurse-generated code. For each partner, the patient had to select the method (SMS/email/postal/gay dating site) and the mode (anonymous/non-anonymous) of sending the notification. All partners - irrespective of the above selected method - received a standardized message with a unique partner code and had to login to the website to read about the notified STI or HIV, possible treatment and how to make an appointment at the STI clinic.

Theoretical framework from the "Technology Acceptance Model" (TAM) was used to develop the questionnaires for index patients and notified partners. ${ }^{10}$ The two factors that determine TAM are "perceived usefulness" (referred to as acceptability) and "perceived ease of use" (referred to as usability). ${ }^{10}$ Questionnaires on acceptability and usability to notify/be notified through Suggestatest.nl of STI and HIV were offered online to all participants regardless their diagnosis/received notification. After the index patient had sent a 
102 Suggestatest.nl notification, an invitation window popped-up to complete an online

103 questionnaire. Partners were recruited for an online questionnaire after reading their STI

104 notification online. After completing the questionnaire, participants were asked to fill-in their

105 email address to receive an additional online questionnaire after 2 weeks. The online

106 questionnaires were collected from March 2012 until June 2013 (Supplementary Tables 1 \&

107 2). Because the online response of partners was low, partners visiting the STI clinics and

108 notified through Suggestatest.nl (who had not yet filled-in an online questionnaire) were

109 recruited from July 2012 until June 2013 to fill in a paper-and-pencil questionnaire.

111 Statistical analysis and data collection

112 All questionnaire data were analyzed in IBM SPSS Statistics version 21 (IBM Corporation,

113 Armonk, New York, USA). The acceptability and usability scores were constructed from the

114 mean of the items included. Constructs were only calculated if none of the items for this

115 construct had a missing values. For each construct, the reliability was calculated using the

116 Spearman-Brown statistic (2-items) or the Cronbach's coefficient alpha ( $\alpha$ ) (3 or more

117 items). ${ }^{11}$ Reliability values of $\geq 0.7$ were assumed acceptable and all were 0.75 or above.

118 Frequency of Internet use for arranging personal matters was categorized in less frequent

119 (scores 1-3) and frequent (scores 4-5). Respondents and non-respondents were compared with

120 the chi-squared test or Fisher's exact test and the Mann-Whitney U test. Using the

121 Independent $t$ test, the mean scores of notified partners who responded to the online and those

122 who responded to the paper-and-pencil questionnaires, were compared. The paired t-test was

123 used to compare scores on different items within the same group. P values of less than 0.05

124 were considered statistically significant.

126 Ethics 
127 This study was waived by the Medical Ethical Committee of the Erasmus University of

128 Rotterdam, because Suggestatest.nl was an extension of standard care.

129 


\section{Index patients}

133 During the study period, $112(19.8 \%)$ out of 565 Suggestatest.nl users completed the

134 questionnaire (Supplementary Figure 1). Response was higher among MSM (27.7\%),

135 compared to heterosexual men $(13.1 \%)$ and women $(17.0 \%, \mathrm{p}=0.002)$, and responders

136 notified a higher median number of partners than non-responders (Supplementary Table 3).

137 Four responders were newly diagnosed with HIV.

139 The majority of index patients reported that they were able to notify more partners than

140 without the existence of Suggestatest.nl (Table 1). The acceptability and usability to use

141 Suggestatest.nl to notify sexual partners of HIV was rated significantly less acceptable and

142 usable (3.0 and 3.6, respectively) than notifying of another STI (4.4 and 4.7, respectively;

$143 \mathrm{p}<0.001$; Table 1). Among MSM, the overall acceptability was higher (4.4) than among non-

144 MSM (4.1; $\mathrm{p}=0.007)$ whereas the overall usability was not different (4.5 versus 4.4,

145 respectively; $\mathrm{p}=0.28$ ).

146

147 Notified partners

148 Out of 2,030 notified partners, $163(8.0 \%)$ responded to the questionnaires (53 online and 110

149 offline at the STI clinic) (Supplementary Figure 1). Notified partners who filled-in the

150 questionnaire were comparable to those who did not respond (Supplementary Table 1). The

151 acceptability and usability scores of online and offline responders were not significantly

152 different. Of the 106 partners who were notified of HIV exposure, three responded to a 153 questionnaire. 
155 Most notified partners preferred to receive a non-anonymous notification via SMS (Table 2).

156 Partners who were notified anonymously rated their notification less acceptable (2.7) than

157 partners who were notified by name $(4.4 ; \mathrm{p}<0.001)$ (Table 2$)$. The acceptability and usability

158 to be notified of HIV through Suggestatest.nl was rated significantly less acceptable and

159 usable (3.3 and 3.2, respectively) than being notified of another STI (both 4.4; $\mathrm{p}<0.001$ ). The

160 overall acceptability and usability scores of Suggestatest.nl (4.1) did not differ between MSM

161 and non-MSM $(\mathrm{p}=0.28$ and $\mathrm{p}=0.50)$.

162

163

164 
Discussion

166

167 Statement of principal findings

168 The online PN tool Suggestatest.nl was rated acceptable and usable by both senders (index

169 patients) and receivers (notified partners). Both groups were less positive about

170 Suggestatest.nl to notify /get notified of HIV than of another STI. Partners notified

171 anonymously perceived their mode of notification less acceptable than those notified by

172 name.

173

174 Strengths and weaknesses of the study

175 While most papers on acceptability of electronic PN relied on hypothetical scenarios, we

176 measured acceptability and usability in a real setting, in both patients and partners who used

177 Suggestatest.nl. ${ }^{9}$ Moreover, we measured the opinion of both MSM and heterosexuals who

178 used Suggestatest.nl. Patients who chose to use Suggestatest.nl may be more enthusiastic

179 about Suggestatest.nl than STI patients in general. However, their partners who did not have

180 any choice in the method of how they received a notification, were also generally positive

181 about Suggestatest.nl.

182

183 For our study, we recruited notified partners when they visited the website to read their

184 notification or during the resulting consultation at the STI clinic. Unfortunately, the overall

185 participation rate of notified partners was low (8\%). This might have resulted in

186 overestimated acceptability and usability scores, making it difficult to generalize the

187 measured opinion to the general STI clinic population. Due to missing notification codes of

18843 notified clients, no information of the received notification was known.

189 
The questions concerning the acceptability and usability of using Suggestatest.nl to notify of

191 HIV exposure were mainly answered by patients and partners who notified or were notified of an STI other than HIV. As a consequence, the lower acceptability and usability to notify of

193 HIV through Suggestatest.nl were mainly hypothetical. Theoretically, the usability to notify

194 partners of STI or HIV exposure through Suggestatest.nl should be comparable because it

195 uses the same system with identical actions. However, the construct of usability was rated

196 lower for HIV than other STI, indicating that it probably did not measure usability only.

\section{Comparison with other studies}

199 A study among Peruvian MSM and transgender women diagnosed with STI showed that the

200 introduction of a hypothetical Internet-based PN system resulted in a dramatic increase in 201 anticipated notification of secondary partners. ${ }^{12}$ In our study, almost $80 \%$ of the index patients reported that they had notified more partners than they would have done without the existence of Suggestatest.nl.

A study among Spanish MSM of their anticipated notification behavior showed that face to

206 face or a phone call were the preferred methods to notify of STI or HIV for both stable and casual partners. ${ }^{13}$ An identifiable SMS was the next most popular method to notify stable and casual partners of STI or HIV. The preference for sending an identifiable SMS contradicts our findings: most patients notified their partners anonymously. ${ }^{6,7}$ A similar effect was seen in a

210 UK study: the preference of respondents for a partner notification method was dependent on 211 whether they see themselves as index patients or contacts. ${ }^{14}$ Another possibility is that 212 patients in our study who were willing to send an identifiable SMS or email have used their 213 own mobile or email and only those with interest in sending an anonymous notification have 214 used Suggestatest.nl. 
216 In a review of the acceptability of electronic PN a pattern emerged across studies showing

217 that anonymity was less acceptable than the electronic delivery method itself. ${ }^{9}$ In our study,

218 the same effect was seen: notified partners were less positive about the fact that their

219 Suggestatest.nl notification was anonymous but were still content about Suggestatest.nl.

\section{Implications for clinicians and policymakers}

222 It seems that, according to the opinion of our patients, STI clinics should offer an online PN tool like Suggestatest.nl. As stated by Hottes et al, a web-based PN service like inSPOT should be supplementary to traditional PN tools. ${ }^{15}$ After developing a PN website, the costs of facilitating online PN are relatively low and it can easily be offered as an addition to already existing traditional tools. Based on our findings we would recommend to incorporate the possibility to notify anonymously.

Patients could be asked to immediately start filling in the contact information of their partners in Suggestatest.nl when they are at the STI clinic for a treatment consultation. Possibly,

231 patients are then more motivated to notify their partners than later at home, and public health nurses could assist with this process. However, it is also important to offer Suggestatest.nl use at a later stage, because at the STI clinic the majority of participants reported that they were unable to fill in contact details of all partners.

\section{Unanswered questions and future research}

237 We recognize that there is a possible trade-off between reaching more partners by the 238 implementation of a low threshold online PN tool and the quality of the sent notification: as 239 many partners do not read their online notification (42\%; e.g. because they think it is an 
240 unsolicited message/spam), the sent notification might not have resulted in health care

241 seeking. ${ }^{6}$ Future research should focus on the most suitable ways of directing online notified

242 partners into care. After the inclusion period of this study, the tool was renamed to

243 "Partnerwaarschuwing.nl" (Partnernotification.nl in English) as some notified partners

244 reported that were confused about the name Suggestatest.nl.

246 Our study mainly focusses on patients who chose to use Suggestatest.nl and their partners in

247 which participation was low. For generalizability, more research is necessary which measures

248 the opinion of all notified STI-clinic clients regarding online partner notification. 
250 MvR, and RK designed the study protocol, supported by HG, TH, and HV. PV was

251 responsible for the development of the Suggestatest.nl website and the implementation of the 252 online questionnaires. MvR performed the statistical analyses supported by $\mathrm{HG}, \mathrm{MvV}$, and 253 HV. MvR, HG and HV drafted the paper, all authors commented on draft versions, and all 254 approved the final version.

Previously presented:

Information from this paper has been disseminated during a poster presentation at the STI \& AIDS World Congress (17 July 2013, Vienna, Austria; abstract number P5.003).

\section{Changed affiliations:}

Maaike G. van Veen: Rutgers, Expert Centre for Sexual and Reproductive Health and Rights,

\section{Correspondence and requests for reprints to:}

265 Martijn van Rooijen, Public Health Service of Amsterdam, Department of Infectious

266 Diseases, Department of Research, P.O. Box 2200, 1000 CE Amsterdam, the Netherlands

267 Tel: +31-20-5555874; Fax: +31-20-5555533.

268 Email: mvrooijen@ggd.amsterdam.nl

\section{Acknowledgements}

271 The authors would like to thank all the nurses working on this project and all the participants

272 of this study. Special thanks goes to Francine van de Heuvel and Mariette Hamers for

273 coordinating the implementation at the STI clinics and distributing the offline sex partner 
274 questionnaires. We would like to thank Udi Davidovich for his suggestions on analysis of the 275 measured questionnaire data.

276 
1. European Centre for Disease Prevention and Control. Public health benefits of partner notification for sexually transmitted infections and HIV. Stockholm: ECDC; 2013. Available at: http://ecdc.europa.eu/en/publications/Publications/Partner-notification-forHIV-STI-June-2013.pdf. Accessed 29 March 2017.

2. Bilardi JE, Fairley CK, Hopkins CA et al. Experiences and outcomes of partner notification among men and women recently diagnosed with Chlamydia and their views on innovative resources aimed at improving notification rates. Sex Transm Dis 2010; 37:253-258.

3. Hopkins CA, Temple-Smith MJ, Fairley CK et al. Telling partners about chlamydia: how acceptable are the new technologies? BMC Infect Dis 2010; 10:58.

4. Reed JL, Huppert JS, Gillespie GL et al. Adolescent patient preferences surrounding partner notification and treatment for sexually transmitted infections. Acad Emerg Med 2015; 22:61-66.

5. Rietmeijer CA, Westergaard B, Mickiewicz TA et al. Evaluation of an online partner notification program. Sex Transm Dis 2011; 38:359-364.

6. Gotz HM, van Rooijen MS, Vriens P et al. Initial evaluation of use of an online partner notification tool for STI, called 'suggest a test': a cross sectional pilot study. Sex Transm Infect 2014; 90:195-200.

7. Correction. Sex Transm Infect 2015; 91:74.

8. Rocha M, Guerreiro R, Pinto N et al. Digital partner notification service at a community-based voluntary counselling and testing centre for men who have sex with men: CheckpointLX, Lisbon, Portugal. International AIDS Conference, At Durban, South Africa 2016; AIDS 2016, Available at: http://programme.aids2016.org/Abstract/Abstract/3702. Accessed 29 March 2017.

9. Pellowski J, Mathews C, Kalichman MO et al. Advancing Partner Notification Through Electronic Communication Technology: A Review of Acceptability and Utilization Research. J Health Commun 2016; 21:629-637.

10. Davis, F. D. "Perceived Usefulness, Perceived Ease of Use, and User Acceptance of Information Technology," MIS Quarterly (13:3), 1989, pp. 319-339.

11. Eisinga R, Grotenhuis M, Pelzer B The reliability of a two-item scale: Pearson, Cronbach, or Spearman-Brown? Int J Public Health 2013; 58:637-642.

12. Clark JL, Segura ER, Perez-Brumer AG et al. Potential impact and acceptability of Internet partner notification for men who have sex with men and transgender women recently diagnosed as having sexually transmitted disease in Lima, Peru. Sex Transm Dis 2014; 41:43-45.

13. Carnicer-Pont D, Barbera-Gracia MJ, Fernandez-Davila P et al. Use of new technologies to notify possible contagion of sexually-transmitted infections among men. Gac Sanit 2015; 29:190-197. 
14. Apoola A, Radcliffe K, Das S et al. Preferences for partner notification method: variation in responses between respondents as index patients and contacts. Int J STD AIDS 2007; 18:493-494.

15. Hottes TS, Gilbert M Evaluation of online partner notification services like inSPOT requires starting with the client, not the clinic. Sex Transm Dis 2012; 39:348. 
Table 1. Acceptability and usability scores and partner notification related answers of index patients who used Suggestatest.nl to notify sex partners, the Netherlands, March 2012 - June 2013

\begin{tabular}{lcc}
\hline & Acceptability & Usability $^{1}$ \\
& $\mathrm{~N}=112$ & mean (sd) \\
\cline { 2 - 3 } & mean (sd) & $4.6(0.7)$ \\
\hline Arrange personal matters via internet & $4.0(0.8)$ & $4.6(0.8)$ \\
Notify sex partners via internet & $4.0(0.9)$ & $4.6(0.7)$ \\
Notify sex partners with SAT while at home & $4.4(1.0)$ & $4.5(0.7)$ \\
Notify with SAT compared to former performed notification & $4.0(1.0)$ & $4.7(0.7)$ \\
method (N=52) & & $3.6(1.4)$ \\
Notify of STI with SAT & \\
Notify of HIV with SAT & \\
The STI clinic offering SAT & $4.4(0.8)$ & $4.8(0.4)$ \\
Willingness to receive notification through SAT & $3.0(1.5)$ & NA $^{\mathbf{4}}$
\end{tabular}




\begin{tabular}{lcc}
\hline & Yes & No \\
& $\mathrm{N}(\%)$ & $59(52.7)$ \\
\hline Experience with notifying partners & $53(47.3)$ & $71(63.4)$ \\
Able to fill in contact information of all partners at the STI clinic & $41(36.6)$ & $88(78.6)$ \\
Notified more partners with Suggestatest.nl than without the & & $24.4)$ \\
existence of SAT
\end{tabular}

NA: not applicable; SAT: Suggestatest.nl; sd: standard deviation

${ }^{1}$ Acceptability and usability scores ranged from 1 to 5 .

${ }^{2}$ As most participants did not notify of HIV, questions about using SAT to notify of STI or HIV exposure were asked regardless of type of notification sent. Four index patients were newly diagnosed with HIV. Three rated Suggestatest.nl as very acceptable and usable to notify partners of both HIV and STI exposure (all scored 5). The other patient was less positive (HIV: 2 and 3.5; STI: 3 and 4, respectively).

${ }^{3}$ This is not based on experience but on the index patient's opinion.

${ }^{4}$ Usability was not applicable for these items because the questionnaires focused on the acceptability of SAT only. 
${ }^{5}$ Overall acceptability and usability are based on all items mentioned in above table except "Notify with SAT compared to former performed notification", because of a relative high number of missing values. 
Table 2. Acceptably and usability scores and partner notification related answers of partners who were notified through Suggestatest.nl, the Netherlands, 01-03-2012 until 31-05-2013

\begin{tabular}{|c|c|c|}
\hline & Acceptability $^{1}$ & Usability $^{1}$ \\
\hline & $\mathrm{N}=163^{2}$ & $\mathrm{~N}=163^{2}$ \\
\hline & mean $(\mathrm{sd})$ & mean $(\mathrm{sd})$ \\
\hline Arrange personal matters via internet & $4.1(0.8)$ & $4.5(0.8)$ \\
\hline Enter a personal code online to view detailed & $4.0(1.2)$ & $4.3(1.1)$ \\
\hline \multicolumn{3}{|l|}{ notification } \\
\hline Read the STI-specific notification using the internet & $4.0(1.0)$ & $4.4(0.9)$ \\
\hline Non-anonymous & $4.4(0.9)$ & $\mathrm{NA}^{4}$ \\
\hline Receive notification via SAT compared to previously & $3.6(1.0)$ & $3.6(1.0)$ \\
\hline \multicolumn{3}{|l|}{ received notification ${ }^{5}$} \\
\hline Receive notification of STI via $\mathrm{SAT}^{6}$ & $4.4(0.9)$ & $4.4(0.9)$ \\
\hline Receive notification of HIV via $\mathrm{SAT}^{6}$ & $3.3(1.5)$ & $3.2(1.5)$ \\
\hline
\end{tabular}


The STI clinic offering SAT

Willingness to send notification through $\mathrm{SAT}^{7}$

Recommend SAT

Overall $^{8}$

Received in the past an STI notification through a

method other than Suggestatest.nl (36 missings)

Yes

No

Preferred method of receiving a notification through

Suggestatest.nl (50 missings)

SMS, anonymous

$31(27.4 \%)$

SMS, non-anonymous

$56(49.6 \%)$

Email, anonymous

$11(9.7 \%)$

Email, non-anonymous

Postal, anonymous
$4.4(0.9)$

$4.1(1.2)$

$4.4(0.9)$

$4.1(0.8)$

$64(50.4 \%)$

$63(49.6 \%)$
$4.5(0.8)$

$\mathrm{NA}^{4}$

$\mathrm{NA}^{4}$

$4.1(0.7)$ 
NA: not applicable; SAT: Suggestatest.nl; sd: standard deviation

${ }^{1}$ Acceptability and usability scores ranged from 1 to 5 .

${ }^{2}$ Total number of questionnaires were $\mathrm{N}=163$ : $\mathrm{N}=53$ were filled in online after sending a notification and $\mathrm{N}=110$ offline when visiting the STI clinic. None of the scores were statistically different between those who responded online and those who responded offline. Due to missing answers, single items and constructs (only calculated if all items were available) were not available for all participants; for acceptability, the items were complete for (from above) 118, 150, 150, 90, 32, 47, 146, 144, 147, 128, 127 and 133 participants respectively; for usability, the items were complete for (from above) 119, 157, 149, 46, 145, 141, 146, and 136 participants respectively.

${ }^{3}$ Opinion about (non)anonymous notification was only measured for the type of received notification (N=90 anonymous, N=32 non-anonymous, $\mathrm{N}=41$ missing).

${ }^{4}$ Usability was not applicable for these items because the questionnaires focused on the acceptability of SAT only.

${ }^{5}$ Only asked to $\mathrm{N}=64$ partners who were notified before. 
${ }^{6}$ Questions on acceptability and usability of SAT to notify for STI and HIV were offered to all participants regardless the type of received notification. Three partners were notified of HIV exposure. They rated Suggestatest.nl as acceptable (mean 4.7; individual scores 4, 5 and 5) and usable (mean 4.2; individual scores 3.5, 4 and 5) to notify of STI. The acceptability and usability to receive an HIV notification through

Suggestatest.nl were rated 4.3 (individual scores 3, 5 and 5) and 3.2 (individual scores 3, 5 and 1.5), respectively.

${ }^{7}$ This is not based on experience but on the opinion of the notified person.

${ }^{8}$ Overall acceptability and usability based on items mentioned in above table except - because of a relative high number of missing values "arrange personal matters via internet", "receive an anonymous or non-anonymous notification", "receive notification via SAT compared to previous received notification", "preference to send notification through SAT", and "recommend SAT". For online respondents, the first item and latter two items were asked only in the follow-up questionnaire participants received 2 weeks after completing the first one ( 23 out of the 53 online responders filled-in). With the latter 2 items included, the mean acceptability score was 4.1 (sd=0.7), and the total number of completed questionnaires was $\mathrm{N}=103$. 
Click here to access/download Supplemental Digital Content suggest a test Accept Usability paper suppl results v3.docx 